\title{
A Discussion on Particle Number and Quantum
}

\section{Indistinguishability}

\author{
Graciela Domenech* And Federico Holik
}

June 14, 2013

Instituto de Astronomía y Física del Espacio (IAFE)

Casilla de Correo 67, Sucursal 28, 1428 Buenos Aires, Argentina

\begin{abstract}
The concept of individuality in quantum mechanics shows radical differences from the concept of individuality in classical physics, as E. Schrödinger pointed out in the early steps of the theory. Regarding this fact, some authors suggested that quantum mechanics does not possess its own language, and therefore, quantum indistinguishability
\end{abstract}

${ }^{*}$ Fellow of the Consejo Nacional de Investigaciones Científicas y Técnicas (CONICET) 
is not incorporated in the theory from the beginning. Nevertheless, it is possible to represent the idea of quantum indistinguishability with a first order language using quasiset theory $(Q)$. In this work, we show that $Q$ cannot capture one of the most important features of quantum non individuality, which is the fact that there are quantum systems for which particle number is not well defined. An axiomatic variant of $Q$, in which quasicardinal is not a primitive concept (for a kind of quasisets called finite quasisets), is also given. This result encourages the searching of theories in which the quasicardinal, being a secondary concept, stands undefined for some quasisets, besides showing explicitly that in a set theory about collections of truly indistinguishable entities, the quasicardinal needs not necessarily be a primitive concept.

Key words: quasisets, particle number, quasicardinality, quantum indistinguishability. 


\section{Introduction}

It is a well established result that the concept of individuality in quantum mechanics clashes radically with its classical counterpart. While in classical physics particles can be considered as individuals without giving rise to consistence problems, in quantum mechanics this is not the case. Contradictions arise if one intends to individuate elementary particles. The responses to this problem range from the claim that there are no elementary particles at all to the assertion that there are particles but they are intrinsically indistinguishable (i.e., indistinguishable in an ontological sense).

The problem is solved in the formalism by imposing symmetrization postulates (in non relativistic quantum mechanics) or, equivalently, by imposing commutation relations on the creation-annihilation operators (in quantum field theory). But these solutions have a flow, well illustrated in [6] and [5]. The objection is that all these approaches make use of a mathematical trick referred to in [6] as the Weyl's strategy. This trick consists in treating particles as if they were individuals and then imposing symmetrization assumptions, thus masking individuality. We find this a source of conceptual confusion and mathematical (or axiomatic) redundancy, for if particles are not individuals (and this implies that they cannot be labeled in the usual way), one could ask why do permutations make sense. Is the symmetrization 
postulate really necessary or is it a necessity of our own (and inadequate) language?

Many authors pointed out the importance of developing alternative ways to describe quantum indistinguishability, reproducing the results obtained by standard techniques, but assuming in every step of the deduction that elementary particles of the same class are intrinsically indistinguishable from the beginning (see, for example, [13], [6] and [5]), without making appeal to Weyl's strategy variants. Another claim is that quantum mechanics does not possess its own language, but it uses a portion of functional analysis which is itself based on set theory, and thus finally related to classical experience. This statement was posed by Y. Manin [8], the Russian mathematician who suggested that standard set theories (as Zermelo-Fraenkel, $Z F$ ) are influenced by every day experience, and so it would be interesting to search for set theories which inspire its concepts in the quantum domain. This is known as the Manin's problem [7]. In this spirit, and looking for a solution to the Manin's problem a quasiset theory ( $Q$ in the following) was developed [6], [15]. We base our work in the axiomatic system as presented in [15].

Quasiset theory seems to be adequate to represent as "sets" of some kind (quasisets) the collections of truly indistinguishable entities. This aim is reached in $Q$ because equality is not a primitive concept, and there exists 
certain kinds of urelemente (m-atoms) for which only an indistinguishability relationship applies. So, in $Q$, non individuality is incorporated by proposing the existence of entities for which it has no sense to assert that they are identical to themselves or different from others of the same class.

$Q$ contains a copy of Zermelo-Fraenkel set theory plus Urelemente $(Z F U)$. These Urelemente are called M-atoms. This feature divides the theory in two parts. One region involves only the elements of $Z F U$, and the other one contains quasisets whose elements can be truly indistinguishable entities. Quasisets containing only indistinguishable elements are called "pure quasisets". We will refer to the $Z F U$ copy of quasisets as "the classical part of the theory", as in [15]. Indistinguishability is modeled in this theory using a primitive binary relation $\equiv$ (indistinguishability) and a new class of atoms, called m-atoms, which stands for expressing the existence of quanta in the theory $([15])$. So, in the frame of $Q$, when we speak of m-atoms of the same class, the only thing that we can assert about them is that they are indistinguishable, and nothing else makes sense, for expressions like $x=y$ are not well formed formulas for them. This is to say that we cannot make assertions about their identity, i.e., it has no sense to say that an m-atom is equal or different of other m-atom of the same class.

It is important to remark that in $Q$, indistinguishability does not imply 
identity, and so it is possible that even being indistinguishable, two m-atoms belong to different quasisets, thus avoiding the collapse of indistinguishability in classical identity [15].

$Q$ is constructed in such a way that allows the existence of collections of truly indistinguishable objects, and thereof it is impossible to label the elements of pure quasisets. For this reason, the construction used to assign cardinals to sets of standard $Z F U$ theories cannot be applied any more.

But even if electrons are indistinguishable (in an ontological sense), every physician knows that it is possible to assert that for example, a Litium atom has three electrons. It is for that reason that $Q$ should allow quasisets to have some kind of associated cardinal. In $Q$ this is solved postulating that a cardinal number is assigned to every quasiset (remember that there is a copy of $Z F U$ in $Q$ ). Some other properties of the standard cardinal are postulated too. This rule for the assignment of cardinals uses a unary symbol $q c()$ as a primitive concept. Here we will call "axioms of quasicardinality" the collection of axioms related to the $q c()$ functional letter. So in $Q$, the quasicardinal is a primitive concept alike $Z F$, in which the property that to every set corresponds a single cardinal number can be derived from the axioms [10].

On the other hand, this form of introducing the quasicardinal implies 
that every quasiset has an associated cardinal, i.e., every quasiset has a well defined number of elements. But the idea that an aggregate of entities must necessarily have an associated number which represents the number of entities is based in our every day experience. As we shall discuss below, (section 2) there are quantum systems for which it is not allowed to assign a number of particles in a consistent manner. These systems can be found in states which are not eigenstates of the particle number operator. Another problematic situation (which will be not discussed in this work) is that of the frame dependence of the particle number operator. In relativistic quantum field theory, the vacuum state in a Minkowskian frame (which has particle number equal to zero in this frame) is seen as a "plenty of particles" state for a Rindler observer [11, thus cardinality seeming frame dependent.

In 2 we will show that from the fact that in $Q$ every quasiset has an associated cardinal (its quasicardinal), it follows that it is not possible to represent systems which are not eigenstates of the particle number operator as quasisets. In 3 we discuss the relationship of the concept of particle number with the measurement process. Based on the discussions previously posed, in 4 we explore the possibility of modifying $Q$ in such a way that the quasicardinal becomes a derived concept. Finally, in 5 we draw our conclusions. 


\section{Quantity in Quantum Mechanics}

As is well known, performing a single measurement in a quantum system does not allow to attribute the result of this measurement to a property which the system possesses before the measurement is performed without giving rise to serious problems [9]. What is the relationship between this fact and the quantity of particles in a quantum system? Take for example an electromagnetic field (with a single frequency for simplicity) in the following state:

$$
|\psi\rangle=\alpha|1\rangle+\beta|2\rangle
$$

where $|1\rangle$ and $|2\rangle$ are eigenvectors of the particle number operator with eigenvalues 1 and 2 respectively, and $\alpha$ and $\beta$ are complex numbers which

satisfy $|\alpha|^{2}+|\beta|^{2}=1$. If a measurement of the number of particles of the system is made, one or two particles will be detected, with probabilities $|\alpha|^{2}$ and $|\beta|^{2}$ respectively. And any other possibility is excluded. Suppose that in a single measurement two particles are detected. What allows us to conclude that the system had two particles before the measurement was performed? The assertion that the number of particles is varying in time because particles are being constantly created and destroyed is also problematic, because it assumes that at each instant the number of particles is 
well defined. Only in case that it is known with certainty that the system is in an eigenstate of the particle number operator we can say that the system has a well defined cardinal. There would be no problem too if it is known with certainty that the system is prepared in an statistical mixture. In this case, the corresponding density operator would be:

$$
\rho_{m}=|\alpha|^{2}|1\rangle\left\langle\left. 1|+| \beta\right|^{2} \mid 2\right\rangle\langle 2|
$$

where the subindex "m" stands for statistical mixture. But the density operator corresponding to (1) is:

$$
\rho=(\alpha|1\rangle+\beta|2\rangle)\left(\alpha^{*}\langle 1|+\beta^{*}\langle 2|\right)
$$

which is the same as:

$$
\rho=|\alpha|^{2}|1\rangle\left\langle\left. 1|+| \beta\right|^{2} \mid 2\right\rangle\left\langle 2\left|+\alpha \beta^{*}\right| 1\right\rangle\left\langle 2\left|+\alpha^{*} \beta\right| 2\right\rangle\langle 1|
$$

The presence of interference terms in the last equation implies that difficulties will appear in stating that, after a single measurement, the system has the quantity of particles obtained as the result of the measurement. In this case, the incapability of knowing the particle number would not come from our ignorance about the system, but from the fact that in this state, the particle number is not even well defined.

Taking into account these considerations, it is worth asking: is it possible to represent a system prepared in the state (40) in the frame of quasiset 
theory? Which place would correspond to a system like (44) in that theory? If such system could be represented as a quasiset, then it should have an associated quasicardinal, for every quasiset has it. But this does not seem to be proper, considering what we have discussed in this section. It follows that it does not appear reasonable to assign a quasicardinal to every quasiset if quasiset theory has to include all bosonic and fermionic systems (in all their possible many particle states). Therefore, a system in the state (4) cannot be included in $Q$ as a quasiset. Yet, it would be interesting to study the possibility of including systems in those states (such as (41)) in the formalism. A possible way out is to reformulate $Q$ in such a way that the quasicardinal is not to be taken as a primitive concept, but as a derived one, turning into a property that some quasisets have and some others do not (in analogy with the property "being a prime number" of the integers). Those quasisets for which the property of having a quasicardinal is not satisfied, would be suitable to represent quantum systems with particle number not defined (such as (44)). This property would also fit well with the position that asserts that particle interpretation is not adequate in, for example, quantum electrodynamics. With such a modification of $Q$, a field (in any state) could always be represented as a quasiset, avoiding the necessity of regarding the field as a collection of classical "things". On the contrary, the 
field would be described by a quasiset which has a defined quasicardinal only in special cases, but not in general. And for that reason this quasiset could not be interpreted as simply as a collection of particles (because it seems reasonable to assume that a collection of particles, indistinguishable or not, must always have a well defined particle number).

Another possibility would be the introduction of a vector space similar to a Hilbert space, but constructed using the non classical part of $Q$. This option will be considered in a forthcoming paper. In the following section we discuss the concept of particle number as the result of a process (the measurement process). We will discuss its relationship with the idea of individuality suggested by experiments, and relate it with the possibility of developing quasicardinal as a derived concept.

\section{Particle number as the result of a process}

In the last section, we suggested that the development of a $Q$-like theory in which quasicardinal is a derived concept could be useful if one aims to represent as quasisets, quantum systems with particle number not defined. In this section, we discuss the experimental relation of the concept of "particle number" and find new arguments for the development of quasicardinal as a derived concept. We start posing the question: In which sense do we talk 
about quantum systems composed, for example, of a single photon? We certainly know about the existence of the electromagnetic field, and that this field obeys the rules of quantum mechanics. How do we decide if the field is in a single photon state or not? What do we mean when we use the words "single photon"? These questions find an answer in our laboratory experience, i.e., making measurements on the system. The measurement process (which in the case of photons could be described by the theory of interaction of the electromagnetic field with matter) allows us to construct an idea of individuality which in time allows us to speak about the photon as a particle. In a similar way, and always mediated by a measuring process, we talk about the other particles (electrons, protons, etc.). But these corpuscular features of quantum systems differ notably from the classical ones, and though experiments suggest an idea of individuality, it is well established that this does not enable us to consider particles as individuals, at least not in an equal sense to classical individuality. Elementary particles cannot be considered as individuals, as E. Schrödinger pointed out in the early days of quantum mechanics [16]. In spite of these difficulties, we continue speaking about photons, electrons, etc., using a jargon which has a lot of points in common with classical physics, source of conceptual confusion.

Let us consider an example to illustrate how particle number arises as 
a result of the measuring process. A photoelectric detector consists in its fundamental aspects of an atom that can be ionized due to the interaction with the electromagnetic field. The signal (a current originated by the ionized atom) must be amplified in order to be detected. The amplified signal is a (macroscopical) current, and we say that the intensity of this current is proportional to the quantity of "absorbed photons" in the volume of the detector (in practice, composed of many atoms). In the limit of single photon states, we would observe a single current pulse each time a photon is detected. Thus, we see that once the detection mechanisms are considered, it is possible to assign to some quantum systems an associated number, which represents the "particle number". It is important to point out that the so called "particle number" only appears, in general, after the measurement process is performed. In other words, experimental experience is the condition for particle interpretation of the corresponding theoretical concepts (for example, of the particle number operator). And we have already mentioned that the measurement process almost always implies the modification of the original state, and that the result of the measurement cannot be attributed in general to a property pertaining to the system before the measure is performed. In particular, it is not true that a particle number can be always assigned in a consistent manner, as we saw in the last section. Thus, 
counting the quantity of elements in quantum mechanics (here understood as measuring particle number) is qualitatively different from counting the quantity of elements of a classical system. In particular, in quantum mechanics the system is usually destroyed or modified when counted, alike the classical case, where the counting process can be made in principle without disturbing the system.

Nevertheless, we know that there exist in nature systems for which it is possible to assign a cardinal in a consistent manner (a well defined number of particles, as for example the electrons of a Litium atom, or single photon states). But they cannot simply be considered as aggregates of individuals as if they were distinguishable. This is to say that there should exist the possibility of counting without distinguishing. If this were not the case, physicians would have never talked about something like "number of indistinguishable particles". Here, the word "counting" is taken in the sense of assigning in a consistent manner a "number of elements" to a system which is not so simple as an individuals aggregate. For example, we could count how many electrons has an Helium atom imagining the following process (perhaps not the best, but possible in principle). Put the atom in a cloud chamber and use radiation to ionize it. Then we would observe the tracks of both, an ion and an electron. It is obvious that the electron track rep- 
resents a system of particle number equal to one and, of course, we cannot ask about the identity of the electron (for it has no identity at all), but the counting process does not depend on this query. The only thing that cares is that we are sure that the track is due to a single electron state, and for that purpose, the identity of the electron does not matter. If we ionize the atom again, we will see the track of a new ion (of charge $2 e$ ), and a new electron track. Which electron is responsible of the second electron track? This query is ill defined, but we still do not care. Now, the counting process has finished, for we cannot extract more electrons. The process finished in two steps, and so we say that an Helium atom has two electrons, and we know that, as the wave function of the electrons is an eigenstate of the particle number operator, no problem of consistence will arise in any other experiment if we make this assertion. In [3] Dalla Chiara and Toraldo di Francia had already noted that we know experimentally that the Helium atom has two electrons, because we can ionize it and extract two separate electrons. They were looking for experimental and theoretical grounds for developing a Quaset theory (for a comparison between $Q$ and Quaset theory see [4]). In the same way, we explore on experimental experience to justify the search of a $Q$-like theory in which quasicardinal is a derived concept.

From the example of the ionized Helium atom, we find that the pro- 
cess of counting the elements of a given "collection" extracting them one by one can be applied to some quantum systems without giving rise to serious contradictions. Then, we should be able of counting the Urelemente of some quasisets too. A radical difference between counting the electrons of an atom and counting the elements in a collection of classical objects in the way shown above is that, in the classical case we can ask about the identity of the extracted element at each step while, in the case of the atom, this cannot be done. But this fact, does not alter the essence of the counting process and we will exploit this fact. In the following section, we will translate this idea to the language of $Q$. As we have already mentioned, $Q$ describes collections of truly indistinguishable objects as quasisets and the quasicardinal is introduced as a primitive concept. The latter is justified arguing that indistinguishability prohibits well ordering, and for that reason the possibility of counting $\grave{a} l a Z F$. We agree that quasisets cannot be counted in the same form as in $Z F$, but our point is that it should be interesting to search for other ways of counting, motivated by physical examples.

Experiments on quantum systems sometimes show corpuscular features, and this suggests an idea of individuality. This idea is a base for developing the concept of particle and subsequently, the notion of particles aggregate. In analogy with this, in the next section we will develop a notion of "indi- 
vidual quasiset", which will be used as a base for developing the notion of quantity of elements of a certain kind of given quasisets. This is to be done without making appeal to classical individuality and avoiding the introduction of quasicardinal as a primitive concept (and avoiding quasicardinality axioms). As in the laboratory, where the system to be counted is submitted to a process which transforms the original state of the system, and only from this modification is that we talk about particle number, the axiomatic variant exposed in the rest of this article submits some quasisets (called finite quasisets) to a process which assigns them a quasicardinal in a consistent manner. We want to consider quasicardinal as the result of a process.

\section{Reformulation of Quasicardinality Axioms}

In section 2 we suggested that it would be interesting to enrich $Q$ in such a way that systems with not defined particle number, could be described with the formalism. We suggested that a possible way to follow is to modify $Q$. To do this, we search for a Q-like theory in which the quasicardinal is not to be taken as a primitive concept, alike $Q$. In such a theory, to have a well defined quasicardinal could be a property that some quasisets possess and others do not, thus allowing the existence of quasisets with their quantity of elements not well defined. In section 3 we discussed the links between the 
concept of particle number and the measurement process. There we recalled that it is possible to assign a particle number in a consistent manner to systems which are not as simply as individual aggregates (for example, to electrons in an atom). We said that this fact suggested that a theory in which quasicardinal is a derived concept could be conceived. In the rest of this section we explore this possibility.

The idea behind the formal construction shown below is very simple. Once we have developed the intuitive idea of a quasiset with one element (the singleton), this is used to count the urelemente of a given quasiset extracting them, one by one, without knowing which one is extracted at each time. The challenge is to express this simple idea in a first order language without identity, and using only the axioms of $Q$ which do not contain the primitive unary functional letter $q c()$. The formal construction shown below enriches the questions posed in [14] about the physical and philosophical implications of the "labeling process" described there, which alike our construction, makes use of the quasicardinality axioms. From now on, we use the same notation as in 15], and also results and definitions therein. 


\subsection{Singletons}

In $Z F$ set theory it is possible to construct, for a particular set, another set whose only element is the given set. This is to say: if $A$ is a set, there exists $\{A\}$, the set whose only element is $A$. Of course, the cardinal of $\{A\}$ is 1 . Its analogous in $Q$ is the quasiset:

$$
[A]={ }_{E}[z: z \equiv A]
$$

the quasiset of all the $z$ indistinguishable from $A .={ }_{E}$ stands for the extensional equality defined in [15]. There, it is defined as a binary relation and it is a derived concept (alike $\equiv$, which is a primitive one). It collapses into classical equality when applied to elements of $Z F U$ or M-atoms, and it is not defined for m-atoms (which are all indistinguishable).

In the frame of $Q$ it is not necessarily true that $[A]$ has a quasicardinal equal to 1 , for it could contain other elements indistinguishable from $A$, (apart from $A$ itself). Our aim in this section is to construct a singleton analogous without using the quasicardinality axioms of quasiset theory (i.e., the axioms listed in [15] which make use of $q c()$ functional letter). To do this, we recall the following construction $([15])$ : suppose that $X$ is such that $X \neq \neq_{E} \emptyset$ and $\exists x(x \in X)$. If $\wp(X)$ stands for the collection of all subquasisets 
of $X$, then there exists the quasiset of the $a \subseteq X$ such that $x \in a$ :

$$
A_{x}={ }_{E}[a \in \wp(X): x \in a]
$$

and therefore

$$
<x>={ }_{E} \cap_{a \in A_{x}} a
$$

exists too. So $\langle x\rangle$ is the intersection of $A_{x}$. Taking this fact into account, it is possible to give the following definitions:

Definition 4.1 Given $X \neq_{E} \emptyset$ and $x \in X$

$$
A_{x}={ }_{E}[a \in \wp(X): x \in a]
$$

Definition 4.2 Given $X \neq_{E} \emptyset$ and $x \in X$

$$
<x>={ }_{E} \cap \cap_{a \in A_{x}} a
$$

is the singleton of $x$ relative to $X$.

In [15], $\langle x\rangle$ is a strong singleton. The interesting point is that we can show that the singleton defined above satisfies the following properties:

Proposition 4.3 In case that $X$ is a set (i. e. an element of the copy of $Z F U)$, if $x \in X$ it follows that

$$
<x>={ }_{E}\{x\}
$$


Proof: Straightforward from the axioms of Q.

In the following proposition, " $Q(X)$ " stands for " $X$ is a quasiset".

Proposition 4.4 Let $X$ be such that $Q(X), x \in X, \alpha$ such that $Q(\alpha)$ and $\alpha \subseteq<x>$. Then

$$
\alpha={ }_{E} \emptyset \vee \alpha={ }_{E}<x>
$$

Proof: $\quad$ There are two possibilities: $x \in \alpha$ or $x \notin \alpha$. Suppose the first holds. From $\alpha \subseteq<x>\subseteq X$, it follows that $\alpha \in \wp(X)$ and if $x \in \alpha$ then $\alpha \in A_{x}$. But in this case, by construction of $\langle x\rangle$, it follows that $\langle x\rangle \subseteq \alpha$ and therefore $\langle x\rangle={ }_{E} \alpha$. On the other hand, if we suppose that $x \notin \alpha$, then: $x \in(<x>\backslash \alpha) \subseteq<x>\subseteq X \longrightarrow(<x>\backslash \alpha) \in A_{x} \longrightarrow<x>\subseteq(<x>\backslash \alpha)$ So we have $\langle x\rangle={ }_{E}(\langle x\rangle \backslash \alpha)$. But if this were true, suppose that $z$ is such that $z \in \alpha$, then $z \in<x>$ and therefore $z$ would belong to $(<x>\backslash \alpha)$ and thus $\neg(z \in \alpha)$, which is a contradiction. For this reason it follows that $\forall z(z \notin \alpha)$ but this is to say that $\alpha={ }_{E} \emptyset$.

The last property states that $\langle x\rangle$ only admits $\emptyset$ and $\langle x\rangle$ itself as subquasisets. This is a suitable property for a quasiset of quasicardinal 1 . But the last assertion has to be taken intuitively for, at this step, we have 
not given a rigorous definition of quasicardinal. Notwithstanding, it is reasonable to interpret singletons as quasisets with only one element, because the last two properties suggest that they are the natural extension of the $Z F$ singleton. In the following sections we will use these intuitive ideas to define the quasicardinal, without making use of the axioms related to the $q c()$ functional letter.

\subsection{Descendant Chains}

Below, we will make use of the following proposition: (were $X \backslash<y>$ stands for the difference between the quasiset $X$ and the singleton $<y>$ )

\section{Proposition 4.5}

$$
\forall X\left(Q(X) \wedge X \neq_{E} \emptyset \longrightarrow \exists y(y \in X \wedge X \backslash<y>\subset X)\right)
$$

Proof: $\quad$ Let us see first that $X \neq_{E} \emptyset \longrightarrow \exists y \in X$. If this were not true, then $\forall y$ we would have $\neg(y \in X)$. But in this case it is easy to convince oneself that $\forall z(z \in X \longleftrightarrow z \in \emptyset)$ and so $X={ }_{E} \emptyset$.

Using that $\exists y(y \in X)$ it is possible to construct $\langle y>$. On the other hand $X \backslash<y>\subseteq X$ by definition of inclusion. But in this case, $y \in X \wedge \neg(y \in$ $X \backslash<y>$ ), so we have $X \backslash<y>\subset X$. 
Taking into account the last proposition, given a non empty quasiset $X$, we can imagine the following process. Let us define first the notion of "direct descendent of $X$ ". If $X \neq \emptyset$ then $\exists z \exists_{Q} Y\left(z \in X \wedge Y=_{E}(X \backslash<y>) \wedge Y \subset\right.$ $X)$ and a quasiset $Y$ with this property will be called a direct descendent of $X$. In the last phrase, " $\exists_{Q} Y$ " stands for "there exists a quasiset $Y$ ".

\section{Definition 4.6}

$$
D D_{X}(Y) \longleftrightarrow \exists z\left(z \in X \wedge Y={ }_{E} X \backslash<z>\right)
$$

$D D_{X}(Y)$ is read as: $Y$ is a direct descendent of $X$.

So, given $X \neq \emptyset$ (by proposition 4.5) there always exists a direct descendent of $X$, let us call it $X^{-}$, which satisfies:

$$
X \supset\left(X^{-}\right)
$$

It could happen that $X^{-}=\emptyset$ or not. If $X^{-} \neq_{E} \emptyset$ it follows that there exists a direct descendent of $X^{-}$, call it $X^{--}$. Then we have:

$$
X \supset X^{-} \supset X^{--}
$$

Going ahead with this process, it could be the case that this chain of inclusions stopped (in case the last quasiset so obtained be the empty quasiset), or that it has no end. So we could conceive two qualitatively different situations: 


\section{Situation 1:}

$$
X \supset X^{-} \supset X^{--} \supset X^{---} \supset \cdots \cdots
$$

(the inclusions chain continues indefinitely)

Situation 2:

$$
X \supset X^{-} \supset X^{--} \supset X^{---} \supset \cdots \cdots \supset \emptyset
$$

(the inclusion chain ends in the empty quasiset).

It is not clear for us how to assure the existence of these chains without making appeal to the quasicardinality axioms. But in the following we will assume that given any arbitrary quasiset, we can always follow this procedure taking into account the example of the Helium atom given in section 3. Suppose we want to describe the collection of the electrons in the atom as a pure quasiset $X$. Then, when the atom is ionized, the free electron track and the electrons in the ion could be interpreted as a singleton $\langle x\rangle$ extracted to $X$, and a quasiset $X^{-}={ }_{E} X \backslash\langle x>$ respectively. If the atom is ionized again, we would obtain another singleton $\langle y\rangle$ extracted to $X^{-}$ and $X^{--}={ }_{E} \emptyset$, with analogous interpretations. Then we obtain the chain of inclusions $X \supset X^{-} \supset \emptyset$ (as in situation 2 described above) which reaches the empty quasiset in two steps, expressing the fact that an Helium atom has two electrons. In the following, we will postulate the existence of these chains. 
To express these ideas in formal terms, it is necessary to translate them to first order language using the axiomatic of $Q$. It is important to remark that in this work, we do not make appeal to the axioms of quasicardinality alike Sant'Anna in [14]. With this aim we make the following definition:

\title{
Definition 4.7
}

\author{
$C D_{X}(\gamma) \longleftrightarrow$ \\ $\left(\gamma \in \wp(\wp(X)) \wedge X \in \gamma \wedge \forall z \forall y\left(z \in \gamma \wedge y \in \gamma \wedge z \neq_{E} y \longrightarrow(z \supset y \vee y \supset z)\right)\right.$ \\ $\left.\wedge \forall z\left(z \in \gamma \wedge z \neq \neq_{E} \emptyset \longrightarrow \exists y\left(y \in \gamma \wedge D D_{z}(y) \wedge \forall w\left(w \in \gamma \wedge D D_{z}(w) \longrightarrow w={ }_{E} y\right)\right)\right)\right)$ \\ $C D_{X}(\gamma)$ is read as: $\gamma$ is a descendant chain of $X$.
}

Definition 4.5 says that $\gamma$ is a descendant chain of $X$ if and only if it is a collection of enclosed subquasisets of $X$ (each one is included in its predecessor) such that if $X \in \gamma, z \in \gamma$ and $z \neq \emptyset$, then there exists a direct descendant of $z$ which belongs to $\gamma$. The definition of descendant chain was made to express in the language of $Q$ (minus quasicardinality axioms) situations 1 and 2 considered above. At the end of this section we will discuss that this concept could also be used to describe another situations.

Using this definition we will introduce the following postulate, which asserts that for any given quasiset there exists at least one descendant chain. 
Axiom 4.8 (Axiom of descendent chains)

$$
\forall_{Q} X\left(X \neq_{E} \emptyset \longrightarrow \exists \gamma\left(C D_{X}(\gamma)\right)\right)
$$

This postulate will be used below to construct the quasicardinal as a derived concept.

\subsection{Finite Quasisets}

In this section, we will introduce the notion of finite quasisets. It is based on the notion of descendant chain given above and involves quasifunctions [15]. We will use the notation $q f(F)$ to express that " $F$ is a quasifunction". A quasifunction is a collection of ordered pairs, and if the pair $\langle x ; y\rangle$ belongs to $F$, we will write $F(x)={ }_{E} y$ whenever $y$ is itself a quasiset. Note that this notation has no sense if $y$ is an $m$-atom, because extensional

equality is not defined for such Urelemente. In the following definition, $\omega$ is the quasiset which contains $\emptyset$ and all its successors [10]. If $n^{+}$is a successor of $n$, then $n^{+}={ }_{E} n \cup\{n\}$.

Definition 4.9 Given $X \neq_{E} \emptyset$ :

$$
\begin{gathered}
\operatorname{Fin}(X) \longleftrightarrow \\
\exists n\left(n \in \omega \wedge \forall \gamma \left(C D _ { X } ( \gamma ) \longrightarrow \exists F \left(F \subseteq \gamma \times n^{+} \wedge q f(F) \wedge\right.\right.\right.
\end{gathered}
$$




$$
\begin{gathered}
<n ; X>\in F \wedge \forall z\left(z \in \gamma \longrightarrow \exists j\left(j \in n^{+} \wedge<j ; z>\in F\right)\right) \wedge \\
\left.\left.\forall j\left(j \in n^{+} \wedge j \neq_{E} 0 \longrightarrow D D_{F(j)}(F(j-1))\right)\right)\right)
\end{gathered}
$$

Fin $(X)$ means: $X$ is finite.

The intuitive interpretation of this definition is that a quasiset will be considered to be finite if and only if all its descendant chains last (reach the empty set) in a finite series of steps. In the following, we will make use of another axiom:

\section{Axiom 4.10}

$$
\begin{gathered}
\forall_{Q} X \forall_{Q} Y(\operatorname{Fin}(X) \wedge \operatorname{Fin}(Y) \wedge Y \equiv X \longrightarrow \\
\left.\left(\left(X \subseteq Y \longrightarrow Y={ }_{E} X\right) \wedge\left(Y \subseteq X \longrightarrow Y={ }_{E} X\right)\right)\right)
\end{gathered}
$$

This postulate means that if a given quasiset is finite, any of its direct descendants is distinguishable from the original quasiset. This is a reasonable assumption, because the only thing that distinguishes two collections of truly indistinguishable objects of the same class should be their quantity of elements. We will define the quasicardinal to fit this intuitive idea. We will need the following proposition:

Proposition 4.11 Let $X$ be a quasiset satisfying $X \neq_{E} \emptyset, \operatorname{Fin}(X)$ y $C D_{X}(\gamma)$. Let $F$ be a quasifuntion as in 4.9. Then $F(0)={ }_{E} \emptyset$ 
Proof: As $F$ is a q-function it follows that there exists $\lambda \in \gamma$ such that $<0 ; \lambda>\in F$. If $\lambda$ were not the empty quasiset then there would exist a direct descendant of $\lambda$, call it $\lambda^{-}$, and this last would pertain to $\gamma$, for $D D_{X}(\gamma)$. By hypothesis, there exists $j \in n^{+}$which satisfies $<j ; \lambda^{-}>\in F$. If $j=0$ then $\left\langle 0 ; \lambda^{-}>\in F \wedge<0 ; \lambda>\in F\right.$. But $\lambda^{-} \subset \lambda$ and so it follows that $\neg\left(\lambda^{-} \equiv \lambda\right)$ (using axiom 4.10) and so $F$ would not be a quasifunction any more. Thereafter $j \neq 0$, and in that case, $j>0$. So $<j ; \lambda^{-}>\in F$ and $<0 ; \lambda>\in F$ by construction of $F$, as $j>0$ it follows that $\lambda \subset \lambda^{-}$. But this is a contradiction. The contradiction comes from the supposition that $\lambda \neq_{E} \emptyset$ and then, we have $<0 ; \emptyset>\in F$. (This is the same that $F(0)=\emptyset$.)

Proposition 4.12 Let $X$ be a non empty and finite quasiset. Then there exists a unique $n$ which satisfies definition 4.9.

Proof: Let us suppose that there are two integers $n$ and $m, n<m$, satisfying definition 4.9. In this case, given a descendant chain $\gamma$, there exist $F$ and $F^{\prime}$ which satisfy:

$$
\begin{gathered}
(a) F(n)={ }_{E} X,(b) F \subseteq \gamma \times n^{+},(c) \forall j\left(D D_{F(j)}(F(j-1))\right) \\
\left(a^{\prime}\right) F^{\prime}(m)={ }_{E} X,\left(b^{\prime}\right) F^{\prime} \subseteq \gamma \times m^{+},\left(c^{\prime}\right) \forall j\left(D D_{F^{\prime}(j)}\left(F^{\prime}(j-1)\right)\right)
\end{gathered}
$$


Moreover, by proposition 4.11 we have that $F(0)={ }_{E} \emptyset$ and $F^{\prime}(0)={ }_{E} \emptyset$ and by hypothesis $m-n>0$. On the other hand,

$$
F(n)={ }_{E} X={ }_{E} F^{\prime}(m) \longrightarrow F(n-1)={ }_{E} X^{-}={ }_{E} F^{\prime}(m-1)
$$

This last is true because there is a single direct descendant of $X$ in $\gamma$ and because of the characteristics of quasifunctions. Due to the fact that this is a process with a finite number of steps, we can continue extracting until 0 is reached, and for that reason, we can arrange the $F(j) \mathrm{s}$ in a table:

$$
\begin{gathered}
F(n)={ }_{E} X={ }_{E} F^{\prime}(m) \\
F(n-1)={ }_{E} X^{-}={ }_{E} F^{\prime}(m-1) \\
\vdots \\
F(0)={ }_{E} F(n-n)={ }_{E} \emptyset={ }_{E} F^{\prime}(m-n)
\end{gathered}
$$

But $j=m-n \neq 0$ implies $F^{\prime}(j)={ }_{E} \emptyset$ with $j \neq 0$ and $F^{\prime}(0)={ }_{E} \emptyset$ (by proposition 4.11). This is absurd because it would imply $\emptyset \subset \emptyset$, and we know that $\emptyset$ cannot be a proper subquasiset of $\emptyset$. The contradiction comes from the supposition that $m-n>0$ and so $m=n$.

The last proposition justifies the following definition: 
Definition 4.13 If $X \neq_{E} \emptyset$ and $X$ is finite, we say that its quasicardinal is the only natural number $n$ according to definition 4.9. Then we write $\operatorname{qcard}(X)=_{E} n$. If $X$ is the empty quasiset, we say that it is finite too and its quasicardinal is zero.

It is important at this point to make the following remark. In the last definition we used the symbol qcard() to express that the quasicardinal of $X$ is $n$. Note the distinction of this derived unary function and the primitive unary function $q c()$ used in [15]. In the next subsection, we show that qcard() has the same properties as $q c()$ when restricted to finite quasisets.

\subsection{Quasicardinality Theorems}

Now, we are ready to prove as theorems all the axioms of quasicardinality of $Q$ for the case of finite quasisets. In the rest of this section, we will assume that all quasisets are finite, unless the contrary is mentioned.

\section{Theorem 4.14}

$$
\forall_{Q} X\left(X \neq_{E} \emptyset \longrightarrow q \operatorname{qard}(X) \neq_{E} \emptyset\right)
$$

Proof:

As $X \neq_{E} \emptyset$, it follows that there exists at least one direct descendant of $X$, and each descendant chain has at least two elements (they surely contain 
$X$ and $\emptyset$ ). So the quasifunctions $F$ are defined starting from $0^{+}$and then, $\operatorname{qcard}(X) \neq_{E} 0$.

\section{Theorem 4.15}

$$
\forall_{Q} X\left(q \operatorname{card}(X)={ }_{E} \alpha \longrightarrow \forall \beta\left(\beta \leq \alpha \longrightarrow \exists_{Q} Y\left(Y \subseteq X \wedge \operatorname{qcard}(Y)={ }_{E} \beta\right)\right)\right)
$$

Proof: Suppose that $q \operatorname{card}(X)={ }_{E} n$ y $m<n$. Let $\gamma$ be a descendant chain of $\mathrm{X}$. So, there exists $F$ such that $F \subseteq \gamma \times n^{+}, F(n)={ }_{E} X$ y $D D_{F(j)}(F(j-1))$. Furthermore, $F(0)={ }_{E} \emptyset$.

Consider $Y={ }_{E} F(m)$. Then $Y \in \gamma$. It is easy to convince oneself that the union of each one of the descendant chains of $\mathrm{Y}$ with the sets that precede $\mathrm{Y}$ in $\gamma$ are descendant chains of $X$. Then, given an arbitrary descendant chain in $\mathrm{Y}$, let us extend it to a descendant chain in $\mathrm{X}$ and restrict the quasifunction corresponding to the last one (according to definition 4.9) to the quasiset which contains $Y$ and its descendants. Then, it follows that $Y$ has quasicardinal $m$.

\section{Theorem 4.16}

$$
\forall_{Q} X \forall_{Q} Y(F i n(X) \wedge X \subset Y \longrightarrow q \operatorname{qard}(X)<\operatorname{qcard}(Y))
$$


Proof: $\quad$ Suppose that $q \operatorname{card}(X) \geq n={ }_{E} \operatorname{qcard}(Y)$. It is clear that:

$$
Y={ }_{E}(Y \backslash X) \cup X
$$

If $X \neq_{E} \emptyset$ it follows that there exists $z$ such that $z \in X$. Then:

$$
Y \backslash<z>={ }_{E}(Y \backslash X) \cup(X \backslash<z>)
$$

or with the usual notation:

$$
Y^{-}={ }_{E}(Y \backslash X) \cup\left(X^{-}\right)
$$

Taking this into account, it is apparent that the descendant chain in $X$ induces a descendant chain in $Y$ :

$$
\begin{aligned}
& X \supset X^{-} \supset X^{--} \supset \cdots \cdots \supset \emptyset \longrightarrow \\
& \longrightarrow Y \supset Y^{-} \supset Y^{--} \supset \cdots \cdots \cdots \cdots
\end{aligned}
$$

with $Y={ }_{E}(Y \backslash X) \cup X, Y^{-}={ }_{E}(Y \backslash X) \cup\left(X^{-}\right), Y^{--}={ }_{E}(Y \backslash X) \cup\left(X^{--}\right)$, etc.

If the empty set is reached in $m={ }_{E} n$ steps, then we obtain:

$$
\emptyset={ }_{E}(Y \backslash X) \cup \emptyset \longrightarrow Y \backslash X={ }_{E} \emptyset \longrightarrow Y={ }_{E} X
$$

but this has no sense. If the empty quasiset is reached in $m>n$ steps, it is equally absurd, because we would arrive at zero in a number of steps greater 
than that needed for any chain of $Y$. So we see that $X$ must be finite too, for if it were not so, it would have a chain in $Y$ with an associated number greater than $n$.

\section{Theorem 4.17}

$$
\begin{gathered}
\forall_{Q} X \forall_{Q} Y(\forall w(w \notin X \vee w \notin Y) \longrightarrow \\
\left(q \operatorname{card}(X \cup Y)={ }_{E} \operatorname{qcard}(X)+q \operatorname{qard}(Y)\right)
\end{gathered}
$$

Proof: $\quad$ First, let us see that the union of finite quasisets gives a finite quasiset. Let $Z={ }_{E} X \cup Y . Z \neq{ }_{E} \emptyset \longrightarrow \exists w(w \in Z) \longrightarrow(w \in X \vee w \in Y)$ but not both. If $w \in X$, we have

$$
Z^{-}={ }_{E} Z \backslash<w>={ }_{E}(X \backslash<w>) \cup Y={ }_{E}\left(X^{-}\right) \cup Y
$$

(if $w$ belongs to $Y$, we obtain an analogous equation). Taking a direct descendent of $Z^{-}$, we obtain one of two possibilities:

$$
Z^{--}={ }_{E}\left(X^{--}\right) \cup Y
$$

or:

$$
Z^{--}={ }_{E}\left(X^{-}\right) \cup\left(Y^{-}\right)
$$

$X$ and $Y$ are finite quasisets, so let us suppose that $X$ has quasicardinal $m$ and that $Y$ has quasicardinal $n$. So all the chains of $X$ vanish in $m$ steps 
and all the chains of $Y$ in $n$ steps. Then, we see that each element in a chain of $Z$, call it $e$, can be expressed as

$$
e={ }_{E} \alpha \cup \beta
$$

where $\alpha$ is an element of some chain in $X$ and $\beta$ in $Y$. Then, any chain in $Z$ vanishes in $m+n$ steps. This fact proves that the quasicardinal of $Z$ is $m+n$. In all these demonstrations, the expressions $n, m$ and $n+m$ steps, have to be associated with the quasifunctions of definition 4.9. We point out once more that we are always dealing with finite processes.

In the following, we will use the following proposition:

Proposition 4.18 Let $X$ be a quasiset such that $q \operatorname{card}(X)={ }_{E} 1$. Then it follows that there exists $z$ satisfying $z \in X$ and $X={ }_{E}<z>$.

Proof: $\quad$ Let $\gamma$ be a chain of $X$. Then there exists a quasifunction $F$ satisfying $F(1)={ }_{E} X$ and (so) $F(0)={ }_{E} \emptyset$. Furthermore, $F$ has the following property: $D D_{F(j)}(F(j-1))$, for any $j \neq_{E} 0$. Then we have $D D_{F(1)}(F(0))$ and for that reason $D D_{X}(\emptyset)$. From this, the existence of $z$ such that $z \in X$ and $X \backslash\left\langle z>={ }_{E} \emptyset\right.$ follows. But in this case, there is no other choice than $X={ }_{E}<z>$. 


\section{Theorem 4.19}

$$
\forall_{Q} X\left(q \operatorname{card}(\wp(X))={ }_{E} 2^{q \operatorname{card}(X)}\right)
$$

Proof: By induction on the quasicardinal. Let us suppose that $X$ has quasicardinal 1. Then, by proposition 4.18 it follows that $\exists z$ with $X={ }_{E}<$ $z>$. Thus, by proposition 4.4, the only possible subquasisets are $\emptyset$ and $X$, i.e., $\langle z\rangle$. It is easy to convince oneself that all the chains of $\wp(X)$ vanish in 2 steps, and therefore it follows that $\operatorname{qcard}(\wp(X))={ }_{E} 2={ }_{E} 2^{q \operatorname{card}(X)}$.

Suppose now than the assumption is true for any quasiset of quasicardinal $n$, and let us see that this is true for $n+1$. Let $X$ be an arbitrary quasiset of quasicardinal $n+1$ and $\gamma$ a chain of this quasiset. Then, it follows that $\operatorname{qcard}\left(X^{-}\right)=_{E} n$, for if $X^{-}$is direct descendant of $X$, we have that there exists $z$ with $z \in X$ and $X={ }_{E} X^{-} \cup<z>$. For this reason:

$$
\operatorname{qcard}(X)={ }_{E} q \operatorname{card}\left(X^{-}\right)+q \operatorname{qard}(<z>) \longrightarrow n+1={ }_{E} q \operatorname{card}\left(X^{-}\right)+1
$$

But in this case we have $q \operatorname{card}\left(P\left(X^{-}\right)\right)={ }_{E} 2^{n}$ and as $P\left(X^{-}\right) \subseteq \wp(X)$ it follows that there exist at least $2^{n}$ different quasisets $\wp(X)$, counting the empty quasiset too. But now we see that there remain $2^{n}$ quasisets formed by the union of each one of the quasisets of $P\left(X^{-}\right)$with $\langle z>$. In this case, we see that $\wp(X)$ has at least $2^{n}+2^{n}={ }_{E} 2^{n+1}$ elements. To see that we have counted all the possible quasisets (all the elements of $\wp(X)$ ) we pro- 
ceed as follows. Let $Y$ be a quasiset such that $Y \in \wp(X)$. Then, only one of two things may happen: $Y \cap<z>={ }_{E} \emptyset$ or $\neg\left(Y \cap<z>={ }_{E} \emptyset\right)$. If the first option is true, it follows that $Y \subseteq X^{-}$and so it has been already counted. If

the second one is true, we have $Y \cap\langle z\rangle={ }_{E}<z>$ (using proposition 4.4), and then $Y={ }_{E} Y \backslash\langle z>\cup\langle z>$ and this is to say that $Y$ is the union of an element of $P\left(X^{-}\right)$with $\langle z>$ and then, it has been already counted. $\square$

\subsection{On the existence of finite quasisets}

We have seen that if there exist finite quasisets in the sense given in this section, these would satisfy the axioms of quasicardinality of $Q$. Now, it is worthwhile to ask: under which conditions do pure and finite quasisets exist?

Assume that there exists a non empty and pure quasiset. Call it $X$. Then, there exists $z$ such that $z \in X$, and its singleton $\langle z\rangle$ exists too. Which is the quasicardinal of $\langle z>$ ? The following theorem gives the answer.

Theorem 4.20 Let $X$ be a non empty quasiset and let $\langle z\rangle$ be a singleton of $X$. Then, $\exists q \operatorname{card}(<z>)$ and $q \operatorname{card}(<z>)={ }_{E} 1$.

Proof: $\quad$ Let $\gamma$ be a descendant chain of $\langle z\rangle$. By proposition 4.4 we 
have that every direct descendant of $\langle z\rangle$ is the empty quasiset. Then the members of $\gamma$ can be shown explicitly: $\gamma={ }_{E}\left[y \in \wp(X): y={ }_{E}<z>\right.$ $\left.\vee y={ }_{E} \emptyset\right]$. So let $F$, be the following quasifunction (expressed as a collection of ordered pairs):

$$
F={ }_{E}[<1 ;<z>>;<0 ; \emptyset>]
$$

$F$ satisfies all the conditions listed in definition 4.9 for $n={ }_{E} 1$. As $\gamma$ is an arbitrary chain, it follows that, by definition of quasicardinal, qcard $(<z>$ )$={ }_{E} 1$

Thus we see that the existence of a pure quasiset with quasicardinal equal to 1 follows directly from the assumption of the existence of some pure non empty quasiset. What do we need to assume for granted the existence of pure quasisets with an arbitrary but finite quasicardinal? To answer this question we first give the following definition:

Definition 4.21 A pure quasiset $X$ is infinite if it is not finite.

If we assume that there exists an infinite quasiset then, we have a "source" of infinite and disjoint singletons, because to be infinite implies that there is at least one descendent chain for which it is not possible to find a quasifunction $F$ as defined in 4.9, But with this arbitrarily big collection of singletons, we can make use of theorem 4.17 as follows. If $\langle z\rangle$ is a singleton 
of the chain and $\langle w\rangle$ is another one which satisfies $\langle z\rangle \cap<w>={ }_{E} \emptyset$, we then have, by theorem 4.17, that:

$$
\operatorname{qcard}(<z>\cup<w>)={ }_{E} \operatorname{qcard}(<z>)+\operatorname{qcard}(<w>)={ }_{E} 1+1={ }_{E} 2
$$

It is obvious that, with this procedure, we can generate finite and pure quasisets with any desired quasicardinal.

\subsection{What do we mean by "counting"?}

What do we mean when we say that a given set in $Z F$ has ten elements? In that theory we say that its cardinal is ten, i.e., it is equivalent to the ordinal number 10. If the set is infinite, we say that it has a cardinal, because we can prove that it can be well ordered, and so it is equivalent to a set of ordinals which has a minimal element (which in turn, is the cardinal of the given set) [10]. This fact lets us assert that every set of $Z F$ has an associated cardinal, and the cardinal is interpreted as the number of elements of the set.

So we see that in $Z F$, the formalized idea of quantity of elements bases itself in the concept of equivalence (the existence of a bijective function between sets). We see that this aspect of the formal construction of cardinality in $Z F$ is in correspondence with the intuitive idea of quantity that we deal in our every day experience: two sets have the same quantity of elements if we can put them in biunivocal correspondence. But we can detach of intu- 
itive interpretations, and say that possessing a cardinal (in the sense of $Z F$ ) is simply a property of the sets deduced from the axioms of $Z F$. Then it is found that the formal development of the theory shows properties which depart radically from our intuition, as the fact that there are many classes of infinity, a non trivial result of set theory [10]. Thus we see how formalism development enriches our concept of infinite, showing properties which are by other means inaccessible to intuitive reasoning.

What happens in $Q$ ? As it can be proved that pure quasisets cannot be well ordered [15], it follows that the argument used in $Z F$ to prove that to every set corresponds a single cardinal no longer holds. This means that in $Q$ we cannot use the same technique as in $Z F$ if we want to assign a quasicardinal to every quasiset in a consistent manner.

Is there any other technique which permits to assign a quasicardinal to any arbitrary given quasiset taking quasicardinal as a derived concept? We have in part given an answer to this query because, as shown in section 4, we can assign a derived quasicardinal to a certain class of quasisets (finite quasisets). This assignment rule is reasonable in the following sense: if it is applied to finite sets (elements of $Z F U$ ), the quasicardinal of definition 4.13 coincides with the one of $Z F$, as can be readily checked out. Furthermore, if we restrict ourselves to the classical part of $Q$, axioms 4.8 and 4.10 are no 
longer needed, for they become theorems of $Z F$ axioms.

Postulates 4.8 and 4.10 exposed in section 4 are interpreted as the translation, to the language of $Q$, of the idea of extracting the elements one by one. But this method is valid only for finite quasisets. The trick of extracting the elements one by one until the empty quasiset is reached fails for every thing which deserves to be called infinite. But we make the following observation: it is possible to find in $Z F$ examples of sets which possess non denumerable descendant chains. So, it happens that the concept of descendant chain is not only applicable to denumerable process (as a first glance may suggest), and this may be useful to extend the definition of quasicardinal to infinite quasisets. Thought we are not prepared to solve the problem now, by taking into account the discussion presented above we can formulate the following problem in $Q$ : it would be interesting to formulate an idea of infinite and pure quasisets (without using the axioms of quasicardinality of $Q)$, and then find a way to compare them. This work would be interesting because the intent to solve it will probably give rise to new techniques (distinct and perhaps inequivalent to the ones known in $Z F$ ) of thinking the infinite, and so, enrich our knowledge of this intricate concept. We think that this is an interesting line of work because obtaining satisfactory results in this direction would be an advance in the development of a generalized 
theory of cardinality. Furthermore, the Manin's problem can be extended to the quest of developing a "set theory" in which some "sets" do not possess an associated cardinal. For the idea that every set must have an associated number of elements is based in our every day experience as well as the idea that its elements have to be individuals.

In this section we have proved that in a theory concerning collections of truly indistinguishable objects (as $Q$ ), the quantity of elements, has not necessarily be taken as a primitive concept. This result encourages the search for a theory in which it is impossible to assign a quasicardinal to certain quasisets in a consistent manner, thus allowing to describe what it seems to happen with some quantum systems, in which non individuality expresses itself in the fact that particle number is not defined, besides ontological indistinguishability. We will explore elsewhere the possibility of using the non classical part of $Q$ without modifying the axioms listed in [15], to make a construction analogous to the state space used in quantum mechanics in order to incorporate undefined particle number systems in the formalism.

\section{Conclusions}

In this work, we have searched for a $Q$-like theory in which the quasicardinal is not taken as a primitive concept alike $Q$. In such a theory, to have 
a well defined quasicardinal would eventually be a property that some quasisets possess, and others do not, thus allowing the existence of quasisets for which their quantity of elements is not well defined. This search was motivated in the discussions given in sections 2 and 3 . For in 2 we discussed the existence of quantum systems with not well defined particle number and studied whether they can be represented as quasisets. We saw that this cannot be done, because every quasiset has a well defined quasicardinal, and suggested that a possible way out is to take quasicardinal as a derived concept. In section [3, we found other motivations for considering quasicardinal as a derived concept. This was done regarding particle number as a result of the measurement process, in the sense that this process may be considered as the basis for the particle number interpretation. In 4 we saw that it is possible to develop a theory about collections of indistinguishable entities in which quasicardinal is not a primitive concept. In doing this, we transcribed to the first order language of $Q$ the idea of counting the elements of a collection extracting their elements one by one, without knowing which is which (because in $Q$ this query is not defined). Thus, we introduced the concept of descendant chains. At the end of the section we discussed the possibility of developing this method to extend the definition to infinite sets.

In the construction shown in 4 we have reobtained that every (finite) 
quasiset has a well defined quasicardinal. Perhaps a deeper transformation in the axiomatic of $Q$ is required to obtain the desired result of undefined quasicardinal. One way would be to explore the possibility of developing not completely defined belonging relations. This is done in Quaset theory (see [3]), but quasicardinal is assumed as a primitive concept there, as in $Q$. Another interesting proposal is to modify logical quantifiers such as " $\exists$ " and " $\forall$ " 2]. The modification could be made in such a way that the property of undefined quasicardinal appear as a product of it.

The axiomatic variant exposed in 4 shows explicitly that in a theory about collections of indistinguishable entities, the quasicardinal needs not be necessarily taken as a primitive concept. This result encourages the research of more complex axiomatic formulations, able to incorporate the quantum systems with undefined particle number as sets of some kind, thus enriching the Manin's problem.

Aknowledgements The authors thank Hector Freytes for useful discussions. They also thank the careful reading of the anonymous referees. F. H. also thanks Newton da Costa and Décio Krause for their interesting commentaries. This work was partially supported by the following grants: PICT 04-17687 (ANPCyT), PIP No 6461/05 (CONICET), UBACyT No X081. 


\section{References}

[1] Ballentine, L., Quantum Mechanics, Prentice Hall Englewood Cliffs, New Jersey (1990).

[2] da Costa, N. C. A. and Krause, D., "Logical and Philosophical Remarks on Quasi-Set Theory", Logic Journal of the Interest Group in Pure and Applied Logics, to appear (2007).

[3] Dalla Chiara, M. L. and Toraldo di Francia, G.: "Identity Questions from Quantum Theory", in Gavroglu, K. et. al., (eds.), Physics, Philosophy and the Scientific Community, Dordrecht, Kluwer Academic Publishers, pp. 39-46 (1995).

[4] Dalla Chiara, M. L., Giuntini, R. and Krause, D., "Quasiset Theories for Microobjects: A Comparision", in Castellani, E. (ed.), Interpreting bodies: Classical and quantum objects in modern physics, Princeton University Press, Princeton (1998).

[5] French, S. and Krause, D., Identity in Physics: A historical, Philosophical, and Formal Analysis, Oxford University Press (2006).

[6] Krause, D., "Why quasi-sets?", Boletim da Sociedade Paranaense de Matematica 20, 73-92 (2003). 
[7] Manin, Yu. I., "Mathematical Problems I: Foundations", in Browder, F. E. (ed.) (1976):, p. 36, cited in [5].

[8] Manin, Yu. I., "A course in mathematical logic", Springer-Verlag, p. 84 (1977), cited in [5].

[9] Mittelstaedt, P., The interpretation of quantum mechanics and the measurement process, Cambridge University Press (1998).

[10] Halmos P., Naive Set Theory, D. Van Nostrand Company (1963).

[11] Halvorson, H. P. and Clifton, R., "Are Rindler quanta real? Inequivalent particle concepts in quantum field theory", British Journal of Philosophy of Science, 0008030, (2000).

[12] Penrose, R., The emperors new mind, p. 32, Oxford Un. Press, (1989).

[13] Post, H., "Individuality and physics", The listener 70, 534-537; reprinted in Vedanta for East and West 32, 14-22. (1963), cited in [5].

[14] Sant'Anna, A., "Labels for non individuals?", Found. Phys. Lett. 18, 0894-9875 (2005). 
[15] Santorelli, A., Krause, D. and Sant'Anna, A., "A critical study on the concept of identity in Zermelo-Fraenkel like axioms and its relationship with quantum statistics", Logique \& Analyse 189-192, 231-260 (2005).

[16] Schrödinger, E., "What is an elementary particle?", reprinted in Castellani, E. (ed.), Interpreting bodies: classical and quantum objects in modern physics, Princeton Un. Press, pp. 197-210, (1998).

[17] Weyl, H., Philosophy of mathematics and natural science, Princeton, Princeton University Press, (1949). 